\title{
Typing and Subtyping Mobility in Boxed Ambients*
}

\author{
Massimo Merro and Vladimiro Sassone \\ University of Sussex
}

\begin{abstract}
We provide a novel type system for Bugliesi et al.'s Boxed Ambients that combines value subtyping with mobility types. The former is based on read/write exchange types, the latter builds on the notion of ambient group. Mobility types allow to specify where an ambient is allowed to stay, closing existing expressiveness gaps in the literature at no additional complexity costs. Subtyping is aimed at achieving maximal generality on both communication and mobility types. We then introduce co-capabilities to express explicit permissions to access ambients. In this setting, ambient types are refined to specify who is allowed to enter an ambient, making a promising framework to model open systems.
\end{abstract}

\section{Introduction}

The calculus of Mobile Ambients, [8], abbreviated MA, is a novel process calculus to describe mobile agents which focuses on three fundamental notions: location awareness [9], mobile computation [4], and local communication. Papers such as $[8,5,7,3]$ demonstrate that MA can describe the run-time behaviour of mobile agents very effectively. In MA, the term $n[P]$ represents an agent, or ambient, named $n$, executing the code $P$. Intuitively an ambient represents a mobile, bounded, and protected space in which a computation takes place.

Ambient names, such as $n$, are used to control access to the ambient computation space and may be dynamically created, as in the $\pi$-calculus [16], by the construct $(\boldsymbol{\nu} n) P$ The ability to move and open ambients is regulated by capabilities that processes possess by prior knowledge or acquire by communication. As an example, the system

$$
k\left[\text { in } n . R_{1} \mid R_{2}\right] \mid n\left[\text { open } k . P \mid m\left[\text { out } n . Q_{1} \mid Q_{2}\right]\right]
$$

contains two ambients, $k$ and $n$, running concurrently. The first, $k$, can migrate to $n$ by virtue of its capability in $n$. The second, $n$, contains a sub-ambient $m[\ldots]$, in addition to the capability open $k$ which allows the opening of any ambient named $k$ if any such ambient exists in the computation space of $n$.

\footnotetext{
* Research supported by 'MyThS: Models and Types for Security in Mobile Distributed Systems', EU FET-GC IST-2001-32617. The first author was funded by EPSRC grant GR/M71169.
} 
Unlike other process calculi, MA focuses primarily on process mobility rather than process communication. As a consequence, the need emerged soon of studying static techniques for constraining the mobility behaviour of ambients. In [6], the authors exhibited a type system to control whether or not an ambient is mobile or may be opened. Subsequently, [5] refined that type system enriching the type of ambient name $n$ with a description of the ambients $n$ may cross. In order to circumvent the need for dependent types, ambients in programmerdefined groups. The type of an ambient is then annotated with the capability of crossing - either from outside, via in, or from inside, via out - the border of ambients of certain groups.

In our opinion, the current mobility types of [5] have some limitations:

$\triangleright$ Types are rather complex, due to the presence of the open capability, which allows ambients to acquire new behaviours by opening contained ambients.

$\triangleright$ No subtyping on mobility has been achieved.

$\triangleright$ The interaction of subjective moves (triggered by a process running inside an ambient) with the open construct tends to produce typings where all ambients are typed mobile. This obliges the authors of [5] to extend MA with objective moves, which move ambients from the outside.

$\triangleright$ An anomaly arises in border-crossing control. Consider the system:

Odysseus[in Horse.out Horse.DESTROY_TROY ] | Horse[in Troy] | Troy[TROJANS]

where Odysseus plans to enter Troy for the well-known deed, with the wellknown method. This system is typeable in [5] under type assumptions of the form: ${ }^{1}$

$$
\begin{aligned}
& \text { Odyseus : amb[Achaean, cross[Toy]] } \\
& \text { Horse }: \operatorname{amb}[\text { Toy, cross }[\text { City] }] \\
& \text { Troy }: \operatorname{amb}[\text { City, }]
\end{aligned}
$$

They express that Odysseus is an ambient of group Achaean which is allowed to cross the boundary of ambients of group Toy; Horse is an ambient of group Toy, which may cross ambients of group City; finally, Troy is an ambient of group City. Suppose now Odysseus moves into the Horse, which subsequently moves into Troy, so that the system evolves to:

\section{Troy[ TROJANS | Horse[Odysseus[out Horse.DESTROY_TROY]]].}

Odysseus may then move out of the Horse and take the TROJANS by surprise whom believed he did not have permission to traverse Troy's walls. $\triangleright$ Ambients cannot determine which agents are allowed to traverse their boundaries.

This paper aims at finding an appropriate formalism that addresses the questions above within the ambient framework. It is well-known that many of the difficulties of MA are caused by the open capability [2]; it thus seems a commendable endeavour to investigate variants of the ambient calculus that drop open.

\footnotetext{
${ }^{1}$ We use simplified types here; in [5] types are more complex.
} 
Boxed Ambients [2], abbreviated BA, is a variant of MA, from which it inherits the primitives in and out with exactly the same semantics. As for communication, BA relies on a completely different model which results from dropping the open capability, and adopting communication across ambient boundaries, between parent and children, similar to those found in the Seal calculus [18]. As pointed out in [2], BA retains much of the expressive power of MA while enhancing the flexibility of typed communication with finer-grained, more effective mechanisms. This makes BA particularly suitable for modelling classical security policies for resource protection and access control. For instance, in [1] $\mathrm{BA}$ is used to model mandatory access control policies within a multilevel security system, including both military security (no read-up, no write-down) and commercial security (no read-up, no write-up).

We introduce a type system to control mobility in BA which profits of the absence of the open capability. As in [5], we use ambient groups to express and enforce properties of names indirectly, as properties of groups of names, but avoiding dependent types. The absence of open simplifies types considerably, as both communication and mobility behaviour of children do not influence the types of their parents. Our types for ambient names consist of four components: the group to which the ambient belongs; a component that describes the mobility constraints of the ambient, viz. where the ambient is allowed to go/reside; one that characterises the communications in which the ambient is involved; and, finally, a set of markers that specify what the ambient name can be used for. Processes and capabilities have similar types, though not identical. In particular, their mobility types express where processes and capabilities may drive to, rather than where they are allowed to stay.

We then equip our type system with a non-trivial form of subtyping which deals with both mobility and communication aspects. Subtyping on mobility is based on the idea that a process can be used wherever processes with a more liberal behaviour (in terms of potential moves) is expected. For communication, generality is again our motor. We employ read/write types which specify separately the type of legal inputs and outputs for processes, and allow a general subsumption rule, so as to push both kinds of subtyping as far as possible. For such a system of inference we prove a set of properties expressing formally the intuitions behind our types. These, together with a subject reduction theorem, make explicit the safety guarantees upheld by the present framework.

Our types for BA meet the first four requirements discussed previously. To go further and attack the last requirement, in $\S 4$ we extend the syntax of BA with co-capabilities, which express explicit permission to traverse boundaries. The idea of introducing co-actions is borrowed from process calculi such as CCS [15] or the $\pi$-calculus [16]. Our co-capabilities, are inspired by [19] and [13], where explicit information about the crossing ambient may be required. Thus, for example, in

$$
m\left[\text { in } n \cdot Q_{1} \mid l\left[\text { out } m \cdot Q_{2}\right]\right]|n[P]| R
$$


$m$ may move into $n$ if $P$ has the form $\overline{\text { in }} m . P_{1} \mid P_{2}$, in which case the system evolves to

$$
n\left[m\left[Q_{1} \mid l\left[\text { out } m . Q_{2}\right]\right]\left|P_{1}\right| P_{2}\right] \mid R
$$

Alternatively, $l$ may emigrate from $m$ if $R$ has the form $\overline{\text { out }} l \cdot R_{1} \mid R_{2}$, and then the system evolves to

$$
m\left[\operatorname{in} n \cdot Q_{1}\right]\left|l\left[Q_{2}\right]\right| n[P]\left|R_{1}\right| R_{2} .
$$

We call the calculus with co-capabilities BSA, for Boxed Safe Ambients. Cocapabilities in BSA are exercised by the target computation by (possibly) naming the ambient allowed to traverse a boundary. In this manner, they allow to enhance ambient types by augmenting the type of $n$ with the set of groups of the ambients allowed to cross $n$ 's boundary. The results for BA are smoothly lifted to BSA. We believe that BSA is a promising framework for the analysis of open systems, because no ambients can be entered - and thus attacked - by an intruder unless the intruder's group name appears in the type of the ambient.

The paper proceeds as follows. In $\S 1$ we review the untyped Boxed Ambients and their standard semantics; $\S 2$ introduces the typed variant of Boxed Ambients and presents the relative subject reduction theorem; $\S 3$ show how our types tackle the Troy's war example above. In $\S 4$, we introduce Boxed Safe Ambients and their typing rules, illustrating their expressiveness again on the example of $\S 3$. The paper ends with a discussion of related works. In this extended abstract all proof are omitted, as is much of the discussion; complete proofs can be found in [14].

\section{The Boxed Ambients}

In this section we review the Boxed Ambient calculus as introduced in [2]. The syntax of processes is given in Table 1 , where $\mathbf{N}$ denotes an infinite set of names. Inactivity, composition, restriction and replication are inherited from mainstream concurrent calculi, most notably the $\pi$-calculus [16]. Specific of the ambient calculus are the ambient construct, $V[P]$, and the prefix via capabilities, $V . P$. Capabilities are obtained from names; given a name $n$, the capability in $n$ allows entry into $n$, the capability out $n$ allows exit out of $n$. Meaningless terms such as in $n[P]$ or $n . P$ are ruled out by the type system in the next section.

Communication is (i) synchronous (though an asynchronous version has been considered [2]); (ii) polyadic, we use boldface when appropriate to represent tuples concisely, as in $(\boldsymbol{x})^{\eta} . P$ and $\langle\boldsymbol{V}\rangle^{\eta} . P$; and, most importantly, (iii) located and across boundaries. Syntactically, located communication is obtained by means of tags specifying the location where the communication has to take place. More precisely, outputs (and similarly inputs) can take one of three forms: (i) $\langle\boldsymbol{V}\rangle^{n} . P$, a message for sub-ambient $n$ from its parent; (ii) $\langle\boldsymbol{V}\rangle^{\uparrow} . P$, a message for the parent from a sub-ambient; (iii) $\langle\boldsymbol{V}\rangle^{\star}$.P , a local communication within the current ambient boundaries. We will omit $\star$ in both $\langle\boldsymbol{V}\rangle^{\star} . P$ and $(\boldsymbol{x})^{\star} . P$, so recovering the usual MA notation. 
Table 1 The Boxed Ambients

Names: $n, m, \ldots, x, y, \ldots \in \mathbf{N}$

Locations:

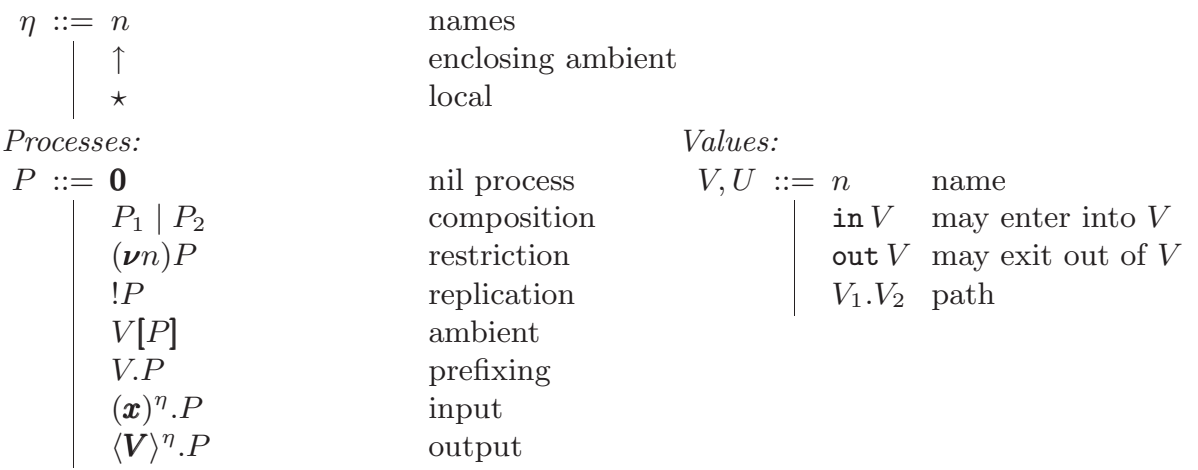

We use a number of notational conventions. Parallel composition has the lowest precedence among the operators. The process $V . V^{\prime} . P$ is read as $V .\left(V^{\prime} . P\right)$. As usual, we omit trailing dead processes, writing $V$ for $V . \mathbf{0},\langle\boldsymbol{V}\rangle$ for $\langle\boldsymbol{V}\rangle . \mathbf{0}$, and $n$ [ ] for $n[\mathbf{0}]$. Restriction $(\boldsymbol{\nu} n) P$ and input prefix $(\boldsymbol{x})^{\eta} . P$ acts as binders for names $n$ and $x$, respectively. The set of free names of $P, f n(P)$, is defined accordingly.

The dynamics of the calculus is given in the form of a reduction relation as in Table 2. As customary in process calculi, the reduction semantics is based on an auxiliary relation called structural congruence, $\equiv$, which brings the participants of a potential interaction to contiguous positions. The reader is referred to [2] for its formal definition. The reduction rules are divided in two groups: the mobility rules and the communication rules. The former are exactly as in MA; the latter add to the usual local communication of MA the location-based communication across boundaries. In the communication rules we assume that tuples have the same arity, a condition that later will be enforced by the type system.

\section{Typing Mobility in Boxed Ambients}

The original types for BA of [2] control that communication is well-typed, wherever it happens. As processes in BA can communicate both locally and upwards, each ambient needs to state explicitly both the local topic of conversation and the topic of the conversation in its parent ambient. There is no need to keep explicit track of communications in the children, as this information is available as the topic of conversation locally in each children.

In this section we extend the types of [2] to control both communication and mobility. The Typed Boxed Ambients are obtained by adding type annotations to the syntax of Table 1 and inheriting the construct $(\boldsymbol{\nu} G) P$ of [5] for group creation. 
Table 2 Reduction Rules

$\rightarrow$ is the least relation on processes closed under ${ }_{-} \mid Q,(\boldsymbol{\nu} n)_{-}, n[-]$ such that: mobility

$$
\begin{aligned}
n[\text { in } m . P \mid Q] \mid m[R] & \rightarrow m[n[P \mid Q] \mid R] \\
m[n[\text { out } m . P \mid Q] \mid R] & \rightarrow n[P \mid Q] \mid m[R]
\end{aligned}
$$

communication

$$
\begin{aligned}
(\boldsymbol{x}) . P \mid\langle\boldsymbol{V}\rangle . Q & \rightarrow P\{\boldsymbol{V} / \boldsymbol{x}\} \mid Q \\
(\boldsymbol{x})^{n} . P \mid n[\langle\boldsymbol{V}\rangle . Q \mid R] & \rightarrow P\{\boldsymbol{V} / \boldsymbol{x}\} \mid n[Q \mid R] \\
(\boldsymbol{x}) . P \mid n\left[\langle\boldsymbol{V}\rangle^{\uparrow} . Q \mid R\right] & \rightarrow P\{\boldsymbol{V} / \boldsymbol{x}\} \mid n[Q \mid R] \\
\langle\boldsymbol{V}\rangle^{n} . P \mid n[(\boldsymbol{x}) . Q \mid R] & \rightarrow P \mid n[Q\{\boldsymbol{V} / \boldsymbol{x}\} \mid R] \\
\langle\boldsymbol{V}\rangle . P \mid n\left[(\boldsymbol{x})^{\uparrow} . Q \mid R\right] & \rightarrow P \mid n[Q\{\boldsymbol{V} / \boldsymbol{x}\} \mid R]
\end{aligned}
$$$$
\text { (RED COMM LOCAL) }
$$

(RED Comm Input $\uparrow$ )

\section{congruence}

$$
P \equiv Q \quad Q \rightarrow R \quad R \equiv S \text { implies } P \rightarrow S \quad \text { (Red Struct) }
$$

The operator $(\boldsymbol{\nu} \mathrm{G}) P$ binds $\mathrm{G}$ in $P$; the set of free groups of $P, \operatorname{fg}(P)$, is defined accordingly. As in [5], groups may appear in the type annotations of both input and name restriction, and the definition of structural congruence must be extended to keep into account the new group operator.

\subsection{The Types}

The type system revolves around three main types: ambient types, process types and capability types; these are all interrelated to each other (actually mutually recursive) and annotated with information on both communication and mobility. A grammar for the types is given in Table 3 .

The first component of ambient types is the group $\mathrm{G}$ which the ambient belongs to. Process (resp. capability) types have a similar component that establishes the group of the ambients where the process (resp. capability) can be executed.

The second component of ambient types describes the mobility constraints within the ambient, expressed in terms of groups rather than actual names. More precisely, in the mobility type mob[S], S represents the (set of groups of) ambients where the ambient in question may reside. As ambients are finally moved by processes, information about mobility must be attached to processes too. Specifically, a process must declare the set of groups of ambients it may drive the enclosing ambient to. For the same reason, since capabilities can be used to form processes, we need to add the same component to capability types as well.

The third component of ambient types disciplines communication along the lines of [2]. In a communication type com $[E, F]$, the exchange type $E$ represents the local topic of conversation, and $F$ the one in the parent. As processes communicate with each other both inside and across ambients, type safety requires 


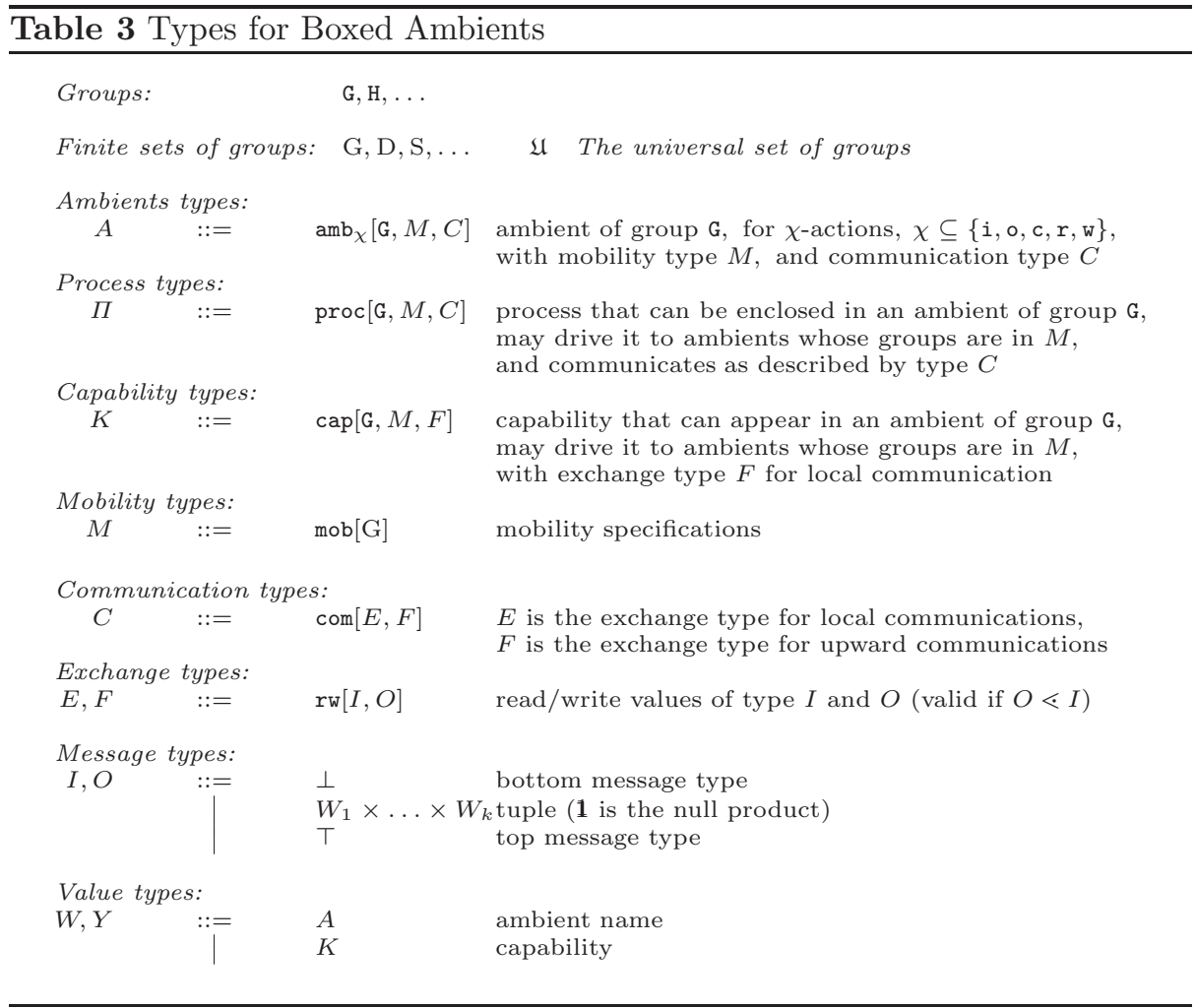

that the exchange of messages must be compatible with the communication type associated to the ambient. It thus follows that processes have a communication type exactly as the above. Things are slightly different for capabilities. Capabilities determine where a process carries its enclosing ambient. Although ambients cannot be opened, the processes they contain can still interact with the receiving ambients by means of upward communication. As a consequence, in order to guarantee safety in mobility, capability types must contain the information about the type of the conversation at destination.

The final component of ambient types - that we denote as an index attached to the keyword $\mathrm{amb}$ - is a set $\chi \subseteq\{\mathrm{i}, \mathrm{o}, \mathrm{c}, \mathrm{r}, \mathrm{w}\}$ which determines the ambient name can be used for, viz. in and out actions, creation of an ambient, reading from and writing to a subambient. This allows to give out names together with restricted capabilities, and refines the capability-passing mechanism of the ambient calculus in that it transmits full knowledge of ambient names, yet selectively releasing rights to act on it. As a matter of notation, we write $\mathrm{amb}_{\text {iow }}$ for $\operatorname{amb}_{\{i, o, w\}}$ and similarly for all $\chi$.

Values in BA consist of tuples of names and capabilities. Since our intention is to have subtyping on values, we must separate the read and write capabilities of exchange types, as initiated in [17]. We do this in a simple, standard way 
Table 4 Subtype Relation

$$
\begin{aligned}
& (\mathrm{sAmB}) \frac{\chi_{1} \subseteq \chi_{0} \subseteq\{\mathrm{i}, \mathrm{o}, \mathrm{c}, \mathrm{r}, \mathrm{w}\}}{\operatorname{amb}_{\chi_{0}}[\mathrm{G}, M, C] \lessdot \mathrm{amb}_{\chi_{1}}[\mathrm{G}, M, C]} \\
& (\mathrm{sCAP}) \frac{M_{0} \lessdot M_{1} ; \quad F_{0} \lessdot F_{1}}{\operatorname{cap}\left[\mathrm{G}, M_{0}, F_{0}\right] \lessdot \operatorname{cap}\left[\mathrm{G}, M_{1}, F_{1}\right]} \\
& (\mathrm{sCoM}) \frac{E_{0} \lessdot E_{1} ; \quad F_{0} \lessdot F_{1}}{\operatorname{com}\left[E_{0}, F_{0}\right] \lessdot \operatorname{com}\left[E_{1}, F_{1}\right]} \\
& (\mathrm{sMsG}) \frac{-}{\perp \lessdot W_{1} \times \ldots \times W_{k} \lessdot \top}
\end{aligned}
$$

(sProc) $\frac{M_{0} \lessdot M_{1} ; \quad C_{0} \lessdot C_{1}}{\operatorname{proc}\left[\mathrm{G}, M_{0}, C_{0}\right] \lessdot \operatorname{proc}\left[\mathrm{G}, M_{1}, C_{1}\right]}$

(sMов) $\frac{\mathrm{G}_{0} \subseteq \mathrm{G}_{1}}{\mathrm{mob}\left[\mathrm{G}_{0}\right] \lessdot \mathrm{mob}\left[\mathrm{G}_{1}\right]}$

$(\operatorname{sExc}) \frac{I_{1} \lessdot I_{0} ; \quad O_{0} \lessdot O_{1}}{\mathrm{rw}\left[I_{0}, O_{0}\right] \lessdot \mathrm{rw}\left[I_{1}, O_{1}\right]}$

(sTuple) $\frac{W_{i} \lessdot Y_{i} ; \quad i \in 1 . . k}{W_{1} \times \ldots \times W_{k} \lessdot Y_{1} \times \ldots \times Y_{k}}$

similar to $[22,20]$. Our exchange types have the form $\mathrm{rw}[I, O]$ and represent the capability of reading and writing values of, respectively, the message types $I$ and $O$. Message types contain tuple types $W_{1} \times \ldots \times W_{k}$ to exchange tuples of values. For $k=0$, the empty tuple type allows the exchange of empty messages, that is, it allows pure synchronisation. Besides tuple types, message types include standard bottom $(\perp)$ and top $(T)$ types that allow to express interesting types. One of these is zero $\triangleq \mathrm{rw}[T, \perp]$, the least exchange type, which describes processes that engage in no communication. As customary with input/output types, we require in $\mathrm{rw}[I, O]$ that $O$ is a subtype of $I$, so that a process output is compatible with (and more specific than) its input. Because of such restriction, that will be assumed throughout the paper, the set of valid types is defined simultaneously with the subtype relation $\lessdot$, whose formal definition is given in Table 4. We will discuss the details in $\S 2.3$ below. For the purposes of this section it suffices to say what follows.

Subtyping on communication allows processes with a communication type $C$ inside ambients with a communication type $C^{\prime}$ if $C \lessdot C^{\prime}$. For instance, a process that exchanges no messages can reside in a ambient regardless of its topic of conversation.

Subtyping on mobility is essentially based on subsets of set of groups, as formalised by rule (sMов). A process driving to ambients with groups in D is allowed to reside in ambients which can move into ambients whose groups belongs to $\mathrm{D}^{\prime}$, with $\mathrm{D} \subseteq \mathrm{D}^{\prime}$.

In $\S 2.3$ we will give a better account about subtyping.

\subsection{Type Assignment}

A type environment $\Gamma$ is a list of assumptions about groups, names and their types of one of two forms: $\mathrm{G}$, declaring the existence of group $\mathrm{G}$, or $n: A$, declaring a name $n$ of type $A$. Since types contain references to groups, the order of assumptions in $\Gamma$ is relevant; to describe valid environments we use judgements of the form $\Gamma \vdash \diamond$ that make sure that names and groups are not repeated 
Table 5 Good Values

$$
(\operatorname{VAL} n) \frac{\Gamma, n: W, \Gamma^{\prime} \vdash \diamond}{\Gamma, n: W, \Gamma^{\prime} \vdash n: W}
$$

$$
\begin{array}{lll}
\text { (VAL PFX) } \frac{\Gamma \vdash V_{0}: K ; \Gamma \vdash V_{1}: K}{\Gamma \vdash V_{0} \cdot V_{1}: K} & \text { (VAL IN) } \frac{\Gamma \vdash V: \operatorname{amb}_{\mathrm{i}}[\mathrm{G}, M, \operatorname{com}[E, F]]}{\Gamma \vdash \operatorname{in} V: \operatorname{cap}[\mathrm{H}, \operatorname{mob}[\{\mathrm{G}\}], E]} \mathrm{H} \in \operatorname{dom}(\Gamma) \\
\text { (VAL SUB) } \frac{\Gamma \vdash V: W ; \quad W \lessdot W^{\prime}}{\Gamma \vdash V: W^{\prime}} & \text { (VAL OUT) } \frac{\Gamma \vdash V: \operatorname{amb}_{0}[\mathrm{G}, M, \operatorname{com}[E, F]]}{\Gamma \vdash \operatorname{out} V: \operatorname{cap}[\mathrm{H}, M, F]} \mathrm{H} \in \operatorname{dom}(\Gamma)
\end{array}
$$

and that groups are defined before being referred to. The formal definition of type environments and domain of an environment, and the inference rules for well-formed environments are standard [5].

Table 5 presents the typing rules for well-formed values. Rules (VAL $n$ ) and (VAL SUB) are straightforward. Rule (VAL PFX) decrees that the type of a path of capabilities is the common type of its components. Notice that, in the light of (VAL SUB) and of the subtyping rule (SCAP) of Table 4, this actually means that paths are assigned a common super type of their component capabilities. In rule (VAL IN), exploiting the subtyping rule (SAMB), we require the in-capability on ambient name $n$ to form in $n$. The conclusion states that the capability in $V$ can reside inside any ambient of group $\mathrm{H}$, for any $\mathrm{H} \in \operatorname{dom}(\Gamma)$, and can drive its enclosing ambient into an ambient of group G (viz. $V$ 's group) with local exchange type $E$ (viz. $V$ 's local exchange type). Rule (VAL OUT) is similar. Its conclusion states that out $V$ can drive the enclosing ambient into ambients with groups mentioned in $M$ (viz. $V$ 's mobility type) and local exchange type $F$ (viz. $V$ 's upward exchange type).

The inference rules in Tables 6 and 7 are for well-typed processes as expressed by judgements of the form $\Gamma \vdash P: \Pi$. We divide the rules in two groups, dealing separately with mobility and communication. Table 6 focuses on mobility. Rule (PRO PFX) builds on the subtyping rules (SCAP) and (SPROC) to state that the characteristics of the capabilities present in a process must be subsumed in its type.

Rule (Pro AmB) is crucial. It says that a process $V[P]$ can be formed only if we have the capability to create an ambient called $V$. Furthermore, $P$ can run inside $V$ only if $P$ and $V$ agree on the group, the mobility type, and the communication type. The resulting ambient $V[P]$ is a process that (i) can be executed in any ambient of group G, provided G is a place where $V$ is allowed to reside; (ii) will not drive an enclosing ambients anywhere; and (iii) inherits $P$ 's upward exchange type as its local exchange type and, finally, has a minimal upward exchange type. As a consequence of the requirement $\mathrm{G} \in \mathrm{S}$, the set $\mathrm{S}$ is never empty; indeed an ambient, even if immobile, always resides somewhere. Notice the role played here by the subtyping rule (SPROC). The remaining rules in Table 6 are straightforward.

Table 7 adapts the rules for communication of [2] taking subtyping into account. 
Table 6 Good Processes - Mobility

$$
\left(\mathrm{PRO} \text { PFX) } \frac{\Gamma \vdash V: \operatorname{cap}[\mathrm{G}, M, F] ; \quad \Gamma \vdash P: \operatorname{proc}[\mathrm{G}, M, \operatorname{com}[E, F]]}{\Gamma \vdash V . P: \operatorname{proc}[\mathrm{G}, M, \operatorname{com}[E, F]]}\right.
$$

(PRO AMB) $\frac{\Gamma \vdash V: \operatorname{amb}_{\mathrm{c}}[\mathrm{H}, \operatorname{mob}[\mathrm{S}], \operatorname{com}[E, F]] ; \Gamma \vdash P: \operatorname{proc}[\mathrm{H}, \operatorname{mob}[\mathrm{S}], \operatorname{com}[E, F]]}{\Gamma \vdash V[P]: \operatorname{proc}[\mathrm{G}, \operatorname{mob}[\varnothing], \operatorname{com}[F, \operatorname{zero}]]} \mathrm{G} \in \mathrm{S}$

(Pro RES) $\frac{\Gamma, n: A \vdash P: \Pi}{\Gamma \vdash(\boldsymbol{\nu} n: A) P: \Pi}$

(PRO GRES) $\frac{\Gamma, \mathrm{G} \vdash P: \Pi}{\Gamma \vdash(\boldsymbol{\nu} \mathrm{G}) P: \Pi} \mathrm{G} \notin \mathrm{fg}(\Pi)$

$\left(\right.$ PRO 0) $\frac{\mathrm{G} \in \operatorname{dom}(\Gamma)}{\Gamma \vdash \mathbf{0}: \operatorname{proc}[\mathrm{G}, \operatorname{mob}[\varnothing], \operatorname{com}[\text { zero, zero }]]}$

(PRO PAR) $\frac{\Gamma \vdash P: \Pi ; \Gamma \vdash Q: \Pi}{\Gamma \vdash P \mid Q: \Pi}$

(PRO REP) $\frac{\Gamma \vdash P: \Pi}{\Gamma \vdash ! P: \Pi}$

(Pro sub) $\frac{\Gamma \vdash P: \Pi \quad \Pi \lessdot \Pi^{\prime}}{\Gamma \vdash P: \Pi^{\prime}}$

\section{Table 7 Good Processes - Communication}

$($ PRO INP $) \frac{\Gamma, x_{1}: W_{1}, \ldots, x_{k}: W_{k} \vdash P: \operatorname{proc}[\mathrm{G}, M, \operatorname{com}[\operatorname{rw}[I, O], F]]}{\Gamma \vdash\left(x_{1}: W_{1}, \ldots, x_{k}: W_{k}\right) \cdot P: \operatorname{proc}[\mathrm{G}, M, \operatorname{com}[\operatorname{rw}[I, O], F]]} \quad I \lessdot W_{1} \times \ldots \times W_{k}$

$(\operatorname{PRO}$ OUT $\star) \frac{\Gamma \vdash V_{1}: W_{1}, \ldots, V_{k}: W_{k} ; \quad \Gamma \vdash P: \operatorname{proc}\left[\mathrm{G}, M, \operatorname{com}\left[\operatorname{rw}\left[I, W_{1} \times \ldots \times W_{k}\right], F\right]\right]}{\Gamma \vdash\left\langle V_{1}, \ldots, V_{k}\right\rangle \cdot P: \operatorname{proc}\left[\mathrm{G}, M, \operatorname{com}\left[\operatorname{rw}\left[I, W_{1} \times \ldots \times W_{k}\right], F\right]\right]}$

(PRO INP $\uparrow) \frac{\Gamma, x_{1}: W_{1}, \ldots, x_{k}: W_{k} \vdash P: \operatorname{proc}[\mathrm{G}, M, \operatorname{com}[E, \operatorname{rw}[I, O]]]}{\Gamma \vdash\left(x_{1}: W_{1}, \ldots, x_{k}: W_{k}\right)^{\uparrow} \cdot P: \operatorname{proc}[\mathrm{G}, M, \operatorname{com}[E, \operatorname{rw}[I, O]]]} \quad I \lessdot W_{1} \times \ldots \times W_{k}$

$(\operatorname{Pro~out~} \uparrow) \frac{\Gamma \vdash V_{1}: W_{1}, \ldots, V_{k}: W_{k} ; \quad \Gamma \vdash P: \operatorname{proc}\left[\mathrm{G}, M, \operatorname{com}\left[E, \operatorname{rw}\left[I, W_{1} \times \ldots \times W_{k}\right]\right]\right]}{\Gamma \vdash\left\langle V_{1}, \ldots, V_{k}\right\rangle^{\uparrow} \cdot P: \operatorname{proc}\left[\mathrm{G}, M, \operatorname{com}\left[E, \operatorname{rw}\left[I, W_{1} \times \ldots \times W_{k}\right]\right]\right]}$

$($ PRO INP $V) \frac{\Gamma \vdash V: \mathrm{amb}_{r}[\mathrm{G}, M, \operatorname{com}[\mathrm{rw}[I, O], F]] ; \Gamma, x_{1}: W_{1}, \ldots, x_{k}: W_{k} \vdash P: \Pi}{\Gamma \vdash\left(x_{1}: W_{1}, \ldots, x_{k}: W_{k}\right)^{V} . P: \Pi} I \lessdot W_{1} \times \ldots \times W_{k}$

$($ Pro out $V) \frac{\Gamma \vdash V: \mathrm{amb}_{\mathrm{w}}\left[\mathrm{G}, M, \operatorname{com}\left[\mathrm{rw}\left[I, W_{k} \times \ldots \times W_{k}\right], F\right]\right] ; \Gamma \vdash U_{1}: W_{1}, \ldots, U_{k}: W_{k} ; \Gamma \vdash P: \Pi}{\Gamma \vdash\left\langle U_{1}, \ldots, U_{k}\right\rangle^{V} . P: \Pi}$

\section{$2.3 \quad$ Subtyping}

Subtyping has a truly relevant impact on type systems for Ambients. Subtyping on communication allows to have processes that exchange no messages in ambients regardless of the local topic of conversation. This idea has been proposed in [22]. In the current paper we put forward the use of subtyping for mobility types, unveiling its beneficial impact and general usefulness for the purpose. The prime idea here is to allow a process that may drive ambients to $\mathrm{D}$ wherever a processes that travels to $\mathrm{D}^{\prime}$, with $\mathrm{D} \subseteq \mathrm{D}^{\prime}$, is expected.

Our aim of fully integrating subtyping in both mobility and communication types is achieved in rules ( $\mathrm{SPROC}$ ) and ( $\mathrm{SCAP}$ ), that state the covariance of process and capability types with their mobility and communication components. Notice that ambient types must be kept invariant, apart from the capabilities $\chi$ that - as typical of these 'may-use-for' situations - follow a contravariant typing. 
Indeed no kind of variance is admissible for mobility or communication, as they both give rise to phony capabilities that trick processes into runtime errors. For instance, if ambient types were to be covariant, their mobility assumptions could be easily defied. Processes might falsely believe that, say, any ambient $n$ at type $\mathrm{amb}_{\chi}\left[-, \mathrm{mob}[\mathrm{S}],{ }_{-}\right]$, with $\mathrm{G} \notin \mathrm{S}$, is allowed to stay in any (ambient of group) G. In this manner, $n$ might end up sitting where it should not. The same net effect is obtained by assuming contravariance, due to the interplay with covariance of process types in their mobility components. Similar arguments can be used to show that ( $\mathrm{SAMB}$ ) must be invariant also in the communication component (details can be found in [14]).

Finally, although in [2] there is no explicit subtyping on names or capabilities, the rules corresponding to our (VAL IN) and (VAL OUT) allow in $n$ and out $n$ an exchange type that is a subtype of the one declared for $n$. This is effectively a form of contravariant subtyping on names. We believe that our approach has advantages over that, as it allows deep, general subtyping on exchange types which, as discussed in [14], constitutes a major difference with the approach of [2].

\subsection{Properties of Typing}

As stated below, our subtype relation satisfies a certain number of properties.

Theorem 1 (Properties of $\lessdot$ ). The subtype relation is a partial order with all bounded joins and meets.

Existing joins $(\sqcup)$ and meets $(\sqcap)$ can be expressed quite easily starting from componentwise set union and intersection for mobility types, e.g., mob[ $\left.\mathrm{S}_{0}\right] \sqcup$ $\operatorname{mob}\left[\mathrm{S}_{1}\right] \triangleq \operatorname{mob}\left[\mathrm{S}_{0} \cup \mathrm{S}_{1}\right]$, from the obvious roles of $\perp$ and $T$ among message types, and from their covariant/contravariant extension to exchange types, that is

$\mathrm{rw}\left[I_{0}, O_{0}\right] \sqcup \mathrm{rw}\left[I_{1}, O_{1}\right] \triangleq \mathrm{rw}\left[I_{0} \sqcap I_{1}, O_{0} \sqcup O_{1}\right] \quad, \quad \mathrm{rw}\left[I_{0}, O_{0}\right] \sqcap \mathrm{rw}\left[I_{1}, O_{1}\right] \triangleq \mathrm{rw}\left[I_{0} \sqcup I_{1}, O_{0} \sqcap O_{1}\right]$.

As a whole, our type systems admits a quite simple algorithmic version - i.e., an equivalent type system with no subsumption rules nor reflexivity and transitivity rules for $\lessdot-$ that helps to prove the following results, intended to formalise the safety guarantees provided by our type system. In the following, we use '-' to avoid naming irrelevant parts of types.

Theorem 2 (Communication Properties). Whenever for some types $W_{1} \times$ $\ldots \times W_{k}$ and $\Pi$ one of the following holds,

$$
\begin{gathered}
\Gamma \vdash\left(x_{1}: W_{1}, \ldots, x_{k}: W_{k}\right) \cdot P \mid\langle\boldsymbol{V}\rangle \cdot Q: \Pi ; \\
\Gamma \vdash\left(x_{1}: W_{1}, \ldots, x_{k}: W_{k}\right) \cdot P\left|m\left[\langle\boldsymbol{V}\rangle^{\uparrow} \cdot Q\right]: \Pi ; \quad \Gamma \vdash\langle\boldsymbol{V}\rangle \cdot Q\right| m\left[\left(x_{1}: W_{1}, \ldots, x_{k}: W_{k}\right)^{\uparrow} \cdot P\right]: \Pi, \\
\Gamma \vdash\left(x_{1}: W_{1}, \ldots, x_{k}: W_{k}\right)^{m} \cdot P\left|m[\langle\boldsymbol{V}\rangle \cdot Q]: \Pi ; \quad \Gamma \vdash\langle\boldsymbol{V}\rangle^{m} \cdot Q\right| m\left[\left(x_{1}: W_{1}, \ldots, x_{k}: W_{k}\right) \cdot P\right]: \Pi
\end{gathered}
$$

then $\boldsymbol{V}=V_{1}, \ldots, V_{k}$ and $\Gamma \vdash V_{1}: Y_{1}, \ldots, V_{k}: Y_{k}$ with $Y_{1} \times \ldots \times Y_{k} \lessdot W_{1} \times$ $\ldots \times W_{k}$. 
Theorem 3 (Mobility Properties). Whenever $\Gamma \vdash n[$ in $m . P \mid Q] \mid m[R]$ : $\Pi$, then

$$
\Gamma \vdash m: \mathrm{amb}_{\chi_{0}}[\mathrm{M},-,-] \text { and } \Gamma \vdash n: \mathrm{amb}_{\chi_{1}}[-, \operatorname{mob}[\mathrm{S}],-]
$$

with $\mathrm{M} \in \mathrm{S}, i, \mathrm{c} \in \chi_{0}$ and $\mathrm{c} \in \chi_{1}$.

Moreover, if for some type $\Pi$ we have $\Gamma \vdash m[n[$ out $m . P \mid Q] \mid R]: \Pi$, then

$$
\Gamma \vdash m: \operatorname{amb}_{\chi_{0}}\left[\mathrm{M}, \operatorname{mob}\left[\mathrm{S}_{m}\right],-\right] \text { and } \Gamma \vdash n: \operatorname{amb}_{\chi_{1}}\left[\mathrm{~N}, \operatorname{mob}\left[\mathrm{S}_{n}\right],-\right]
$$

with $\circ, \mathrm{c} \in \chi_{0}, \mathrm{c} \in \chi_{1}, \mathrm{M} \in \mathrm{S}_{n}$ and $\mathrm{S}_{m} \subseteq \mathrm{S}_{n}$.

Theorem 4 (Subject Congruence and Reduction). If $\Gamma \vdash P: \Pi$ and $P \equiv Q$ or $P \rightarrow Q$, then there exist groups $\mathrm{G}_{0}, \ldots \mathrm{G}_{k}$ such that $\mathrm{G}_{0}, \ldots, \mathrm{G}_{k}, \Gamma \vdash$ $Q: \Pi$.

\section{Some MyThS}

As pointed out in the introduction, the system

Odysseus[in Horse.out Horse.DESTROY_TROY] | Horse[in Troy] | Troy[TROJANS]

can be typed with the types of [5] under hypotheses that say very little about the intentions of Odysseus to traverse Troy's walls or indeed about the permission he might hold for doing so. On the contrary, in our system the term above can be only typed under assumptions of the kind

$$
\begin{aligned}
& \text { Odysseus : } \operatorname{amb}_{c}[\text { Achaean, mob }[\{\text { Ground, Toy, City }\}],] \\
& \text { Horse }: \operatorname{amb}_{\text {ioc }}[\text { Toy, mob }[\{\text { Ground, City }\}],-] \\
& \text { Troy }: \operatorname{amb}_{i o c}[\text { City, _, }]
\end{aligned}
$$

that make explicit the hypotheses that Odysseus is an Achaean intentioned to move into a City. Such information should be enough to alert the TROJANS about an attack on Troy by Odysseus. On the other hand, under assumptions of the form

$$
\text { Odysseus : } \operatorname{amb}_{c}[\text { Achaean, } \operatorname{mob}[\{\text { Ground, Toy }\}],]
$$

the TROJANS should not fear any attack from Odysseus.

So far so good. Provided the TROJANS are in a position of believing Odysseus's declared intentions of just 'moving into some Toy' everything is in place. But what if they suspect that the wily Odysseus might be lying about his real intentions, that is about his real type? The situation would then be perfectly analogous to what happens in the real-world of open-ended systems, where we cannot ask - nor afford - to type-check all systems which we interact with and, at the same time, we cannot trust their declarations of goodwill. What precautions can be taken to avoid being attacked by a malicious agent? What precautions can the TROJANS take to avoid Odysseus's ravage?

A first solution is to have a type inference algorithm to infer a proper type for the external code. Then, analysing the mobility type of Odysseus, Troy can 
check whether its enemy is intentioned to move in. A more robust alternative is to provide Troy with a tool to explicitly declare the ambients allowed to move in. For instance, one may extend the mobility types with a second component that records the groups of those agents which are allowed to move into the ambient (in this case Troy). This would permit checking in movements by simply testing whether the moving ambients is accepted at destination. Unfortunately, we could not do the same test for out actions because target ambients in out movements may vary at run-time.

We avoid the obstacle by introducing the Boxed Safe Ambients a variant of BA enriched with co-capabilities to express permissions to move into ambients.

\section{Safe Boxed Ambients}

The Boxed Safe Ambients, abbreviated BSA, are obtained by extending the definition of Values of Table 1 as follows:

$$
\begin{aligned}
& V::=\ldots \quad \text { as in Typed Boxed Ambients } \\
& \text { in } \alpha \quad \alpha \in\{n, \star\} \quad \text { allow enter of } n \text { or of all } \\
& \overline{\text { out }} \alpha \quad \alpha \in\{n, \star\} \quad \text { allow exit of } n \text { or of all }
\end{aligned}
$$

Using co-capabilities, a BSA ambient can discriminate which ambient groups are allowed to enter its computation space, and when. The co-capabilities of BSA, inspired by $[12,13,19]$, are a novel combination of existing proposals. In particular, both $\overline{\mathrm{i} n}$ and $\overline{\mathrm{out}}$ co-capabilities are placed in the target computation, as in [13]. However, unlike [13], we allow both explicit and anonymous cocapabilities as in [19] and [12], respectively. The former indicates explicitly the name of the ambient allowed to cross the ambient boundary; the latter represents a general permission to cross the boundary usable by all.

The reduction relation is obtained by replacing the rules (Red In) and (Red Out) of Table 2 with the following rules.

$$
\begin{array}{rlll}
n[\operatorname{in} m . P \mid Q] \mid m[\text { in } \alpha . R \mid S] & \rightarrow m[n[P \mid Q]|R| S] & \text { for } \alpha \in\{\star, n\} & \text { (Red IN) } \\
m[n[\text { out } m . P \mid Q] \mid R] \mid \overline{\text { out }} \alpha . S & \rightarrow n[P \mid Q]|m[R]| S & \text { for } \alpha \in\{\star, n\} & \text { (Red Out) }
\end{array}
$$

The types for BSA are obtained by enriching the mobility types of Table 3 with an extra information about the groups of ambients allowed to stay in the given ambient:

$$
M \quad:=\operatorname{mob}[\mathrm{S}, \mathrm{C}] .
$$

The subtyping rules of Table 4 are adapted by replacing the rule (sMoB) with:

$$
\text { ( } \mathrm{sMoB}) \frac{\mathrm{G}_{0} \subseteq \mathrm{G}_{1}, \quad \mathrm{C}_{0} \subseteq \mathrm{C}_{1}}{\mathrm{mob}\left[\mathrm{G}_{0}, \mathrm{C}_{0}\right] \lessdot \mathrm{mob}\left[\mathrm{G}_{1}, \mathrm{C}_{1}\right]} .
$$

The inference rules for well-typed values of Table 5 must be adapted to take into account the new component of the capability types, as in Table 8. Rules (VAL in $V$ ) and (VAL out $V$ ) are like the corresponding ones in Table 5. 


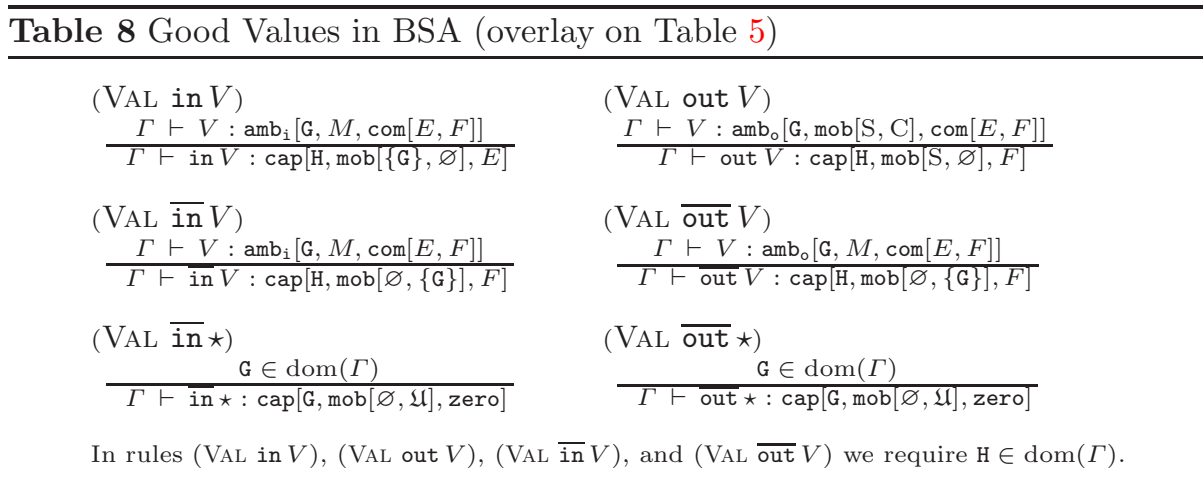

Table 9 Good Processes - Mobility (overlay on Table 6)

(PRO PFX)

$\frac{\Gamma \vdash V: \operatorname{cap}[\mathrm{G}, M, F] ; \quad \Gamma \vdash P: \operatorname{proc}[\mathrm{G}, M, \operatorname{com}[E, F]]}{\Gamma \vdash V \cdot P: \operatorname{proc}[\mathrm{G}, M, \operatorname{com}[E, F]]}$

(PRo 0)

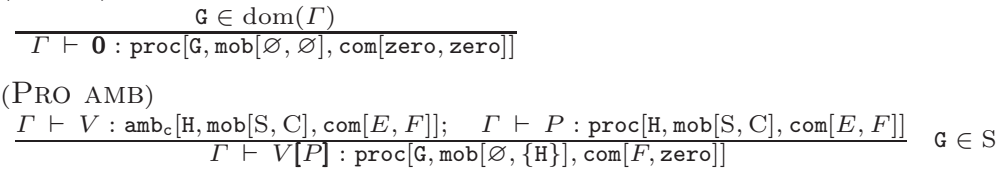

We simply add a second empty component to the mobility type, denoting that these capabilities allow no incoming code. Dually, rules (VAL in $V$ ) and (VAL $\overline{\text { out }} V$ ) do not trigger movements, but allow inbound code. Thus, the resulting mobility types in both conclusions have the first component empty, while the second one record the groups of possible visitors (i.e. the group of $V$ ). The third component of both capability types is the upward exchange type of $V$. As for rules (VAL $\overline{\text { in }} \star$ ) and (VAL $\overline{\text { out }} \star$ ), they differ from the previous ones in the second component of the mobility types where the universal set of groups $\mathfrak{U}$ is used to reflect the anonymous nature of the construct. Moreover, in both cases the exchange type is zero, the least exchange type, because nothing is known about the communication types of the incoming ambients.

The inference rules for well-typed processes are changed as in Table 9; the remaining rules are changed in the obvious manner.

Our results extends smoothly to BSA. In particular, the Subject Reduction Theorem 4 can be restated in formally identical terms for BSA as for BA. The new results concern the added control power granted to BSA by co-capabilities.

Theorem 5 (Control Properties). In addition to the properties of Theorems 2 and 3, whenever

$$
\Gamma \vdash m[\overline{\text { in }} \alpha . P \mid Q]: \Pi \quad \text { or } \quad \Gamma \vdash m[\overline{\text { out }} \alpha . P \mid Q]: \Pi \quad \text { with } \alpha \in\{\star, n\},
$$


then $\Gamma \vdash m: \mathrm{amb}_{\chi_{0}}[-, \operatorname{mob}[-, \mathrm{C}],-]$ and

- either $\alpha=\star$ and $\mathrm{C}=\mathfrak{U}$,

- or $\alpha=n$ with $\Gamma \vdash n: \operatorname{amb}_{\chi_{1}}\left[\mathrm{~N}_{-},{ }_{-}\right]$and $\mathrm{N} \in \mathrm{C}$.

\subsection{Using Co-capabilities to Defend Troy}

The system discussed in $\S 3$ can be rewritten in BSA as follows.

$$
\begin{aligned}
\text { THE_TROJAN_WAR } \triangleq & \text { Odysseus[in Horse.out Horse.DESTROY_TROY] } \\
& \mid \text { Horse }[\overline{\text { in }} \star \text {.in Troy }] \\
& \mid \text { Troy[in Horse.TROJANS | out Odysseus.SINON] }
\end{aligned}
$$

Here, although the TROJANS let the Horse inside the city walls, Odysseus still needs a co-capability, such as out Odysseus executed by SINON from Troy, to be able to get out of the Horse. However, a behaviour like SINON's from a process running in Troy can only be well-typed if Troy has a type allowing ambients of group Achaean to enter Troy, as for instance the following one.

$$
\text { Troy : } \left.\operatorname{amb}_{\text {ioc }}[\text { City, mob[-, }\{\text { Toy, Achaean }\}],-\right]
$$

Of course, such a choice would be suicidal for Troy!

On the other hand, consider the system below, where we remove the security breach represented by SINON.

$$
\begin{aligned}
\text { THE_TROJAN_TRAP } \triangleq & \text { Odysseus[in Horse.out Horse.DESTROY_TROY] } \\
& \mid \text { Horse }[\overline{\text { in }} \star \text {.in Troy }] \mid \text { Troy }[\overline{\text { in } H o r s e . T R O J A N S ~}]
\end{aligned}
$$

This situation would be perfectly safe for Troy (but dangerous for Odysseus!) provided we can type it under the assumptions of the form

$$
\begin{aligned}
& \text { Odysseus : } \operatorname{amb}_{\mathrm{c}}[\text { Achaean, },,] \\
& \text { Horse }: \operatorname{amb}_{\text {ioc }}[\text { Toy, }, \operatorname{com}[E, \text { zero]] } \\
& \text { Troy : } \operatorname{amb}_{\text {ioc }}[\text { City, } \operatorname{mob}[\varnothing, \mathrm{C}],-]
\end{aligned}
$$

with Achaean $\notin \mathrm{C}$. In fact, even though the Horse gets inside the walls no TROJANS will ever give Odysseus permission to get out of it (and he will be stuck to starvation inside it), as assured by the condition for Achaean $\notin$ C. Furthermore, the upward exchange type of the Horse prevents leakage of information from Troy.

\section{Conclusion and Related Work}

We have presented a powerful type system to control communication and mobility within Bugliesi et al.'s Boxed Ambients. A major feature of our approach is the extensive use of a subtyping relation well integrated and crucially relied 
upon within the system. Our type system as a whole enjoys the expected property of subject reduction and provides strong safety guarantees for well-typed terms. These results are formally stated in $\S 2$ and $\S 4$ and exemplified in $\S 3$. We are currently investigating type inference algorithms.

Our types and subtyping compare well with related proposals in the literature. In particular, while the treatment of exchange types is similar to Zimmer's [22] and Yoshida and Hennessy's [20] - in turn elaborations over Pierce and Sangiorgi's input/output types [17] - we know of no similar approaches to mobility types. Work of Yoshida and Hennessy's [21] and Castagna et al.'s [11] use subtyping on interface types for processes of, respectively, a higher-order $\pi$ calculus and the Seal calculus. These approaches, however, are not directly concerned with control of subjective mobility. On the contrary, De Nicola et al.'s [10] focuses on mobility control using subtyping over process capabilities, but for a Linda-like language rather far from ours.

In the second part of the paper we extended the Boxed Ambients with a version of co-capabilities where the entering/exiting ambient can, though need not, be explicitly mentioned. The information gathered in ambient types, together with the power granted to the calculus by co-capabilities, allow to express properties not expressible in previous frameworks. In particular, they permit to deal with situations typical of open-ended systems, as we exemplified by means of the Troy's War example. We believe that our approach here is totally compatible with the moded types of [2] and plan in future work to investigate the matter further.

Finally, note that within our type theory it is possible to analyse immobility properties of ambients. Namely, $n[P]$ is immobile when $P$ can be assigned a type of the form proc[-, mob $[\varnothing]$, _]. However, prescribing that an ambient cannot be moved is a different matter. Recall that the mobility component of an ambient type describes where ambients are allowed to stay, and not to travel to, as for process types. Assigning mob $[\varnothing]$ as mobility type will therefore not help. (As a matter of fact, and as revealed by inspection of rule (PRO AMB), S can never be empty.) This is, we believe, a minor point that can be handled on top of the existing framework with minimal changes. For instance, it would be enough to think of the set of group names as partitioned in two infinite subsets: the mobile and the immobile groups. Then, we only need to add to (VAL IN) and (VAL OUT) the side condition that $\mathrm{H}$ is mobile. Ambients could then be declared immobile by assigning them - either in $\Gamma$ or in a name declarations - to an immobile group.

\section{References}

1. M. Bugliesi, G. Castagna and S. Crafa. Reasoning about security in mobile ambients. In Proc. CONCUR 2001, volume 2154 Lecture Notes in Computer Science, Springer, 2001. 306

2. M. Bugliesi, G. Castagna, and S. Crafa. Boxed ambients. In Proc. TACS 2001, volume 2215 of Lecture Notes in Computer Science, Springer, 2001. 305, 306, 307, 308, 309, 312, 314, 319 
3. L. Cardelli and G. Ghelli. A query language based an the ambient logic. In Proc. ESOP 2001, volume 2028of Lecture Notes in Computer Science, Springer, 2001. 304

4. L. Cardelli. Wide area computation, In Proc. ICALP 1999, volume 1644 of Lecture Notes in Computer Science, Springer, 1999. 304

5. L. Cardelli, G. Ghelli, and A. Gordon. Ambient groups and mobility types. In Proc. IFIP TCS 2000, volume 1872 of Lecture Notes in Computer Science, Springer, 2000. 304, 305, 306, 308, 309, 312, 315

6. L. Cardelli and A. Gordon. Types for mobile ambients. In Proc. of POPL'99, ACM Press, 1999. 305

7. L. Cardelli and A. Gordon. Anytime, anywhere: Modal logics for mobile ambients. In Proc. of POPL 2000, ACM Press, 2000. 304

8. L. Cardelli and A. Gordon. Mobile nmbients. Theoretical Computer Science, 240(1):177213, 2000. An extended abstract appeared in Proc. of FoSSaCS 1998, volume 1378 of Lecture Notes in Computer Science, Springer, 1998. 304

9. I. Castellani. Process algebras with localities. In J. Bergstra, A. Ponse, and S. Smolka, (Eds), Handbook of Process Algebra, 945-1045, North-Holland, 2001. 304

10. R. De Nicola, G. Ferrari, and R. Pugliese. Klaim: A kernel language for agents interaction und mobility. IEEE Trans. an Software Engineering, 24(5), IEEE Press, 1998. 319

11. G. Ghelli G. Castagna and F. Zappa Nardelli. Typing mobility in the seal calculus. In Proc. CONCUR 2001, volume 2154 Lecture Notes in Computer Science, Springer, 2001. 319

12. E Levi and D. Sangiorgi. Controlling interference in ambients. In Proc. POPL 2000, ACM Press. 316

13. M. Merro and M. Hennessy. Bisimulation congruences in safe ambients. Proc. POPL'02, ACM Press, 2002 306, 316

14. M. Merro and V Sassone. Typing and subtyping mobility in boxed ambients. To appear as Technical Report available at http://www.cogs.susx.ac.uk/reports. University of Sussex. 307, 314

15. R. Milner. Communication und Concurrency. Prentice Hall, 1989. 306

16. R. Milner, J. Parrow, and D. Walken A calculus of mobile processes, (Parts I und II). Information and Computation, 100:1-77, 1992. 304, 306, 307

17. B. Pierce and D. Sangiorgi. Typing und subtyping for mobile processes. Journal of Mathematical Structures in Computer Science, 6(5):409-454, 1996. 310, 319

18. J. Vitek and G. Castagna. Seal: A framework for secure mobile computations. In Internet Programming Languages, volume 1686 of Lecture Notes in Computer Science, Springer, 1999. 306

19. Y Yang, X. Guan, and J. You. Typing evolving nmbients. Information Processing Letters, 80(5):265-270, 2001. 306, 316

20. N. Yoshida and M. Hennessy. Subtyping and locality in distributed higher order processes. In Proc. CONCUR 1999, volume 1664 of Lecture Notes in Computer Science, Springer, 1999. 311, 319

21. N. Yoshida and M. Hennessy. Assigning types to processes. In Proc. LICS 2000, IEEE Press, 2000. 319

22. P. Zimmer. Subtyping and typing algorithms for mobile ambients. In Proc. FoSSaCS 2000, volume 1784 of Lecture Notes in Computer Science, Springer, 2000. 311, 313, 319 